\title{
Factores asociados a Hemorragia Intraventricular en Neonatos Prematuros en el Hospital Regional Docente de Trujillo. Diciembre 20 I I a Diciembre 20 I 3.
}

\author{
Glauco Valdivieso ${ }^{1}$, Juan C. Ramírez ${ }^{2}$
}

\section{RESUMEN}

Objetivo: Determinar los factores asociados a hemorragia intraventricular en neonatos prematuros en el Hospital Regional Docente de Trujillo en los periodos Diciembre 2011 a Diciembre 2013.

Material y Métodos: Se llevó a cabo un estudio analítico, retrospectivo de casos y controles. La población estuvo constituida por 108 pacientes: 54 neonatos prematuros con Hemorragia intraventricular y 54 neonatos prematuros sin Hemorragia intraventricular.

Resultados: El peso al nacer resultó ser significativo (x2: 6.438; valor p: 0.011) obteniendo mayor frecuencia de peso menor o igual a $1500 \mathrm{~g}$. El sexo del recién nacido resultó ser una variable significativa (x2: 6.366; valor p: 0.012) siendo en su mayoría el sexo masculino. La edad gestacional resultó ser una variable significativa ( 2 2: 10.394; valor p: 0.001$)$ siendo más frecuente la edad menor o igual a 32 semanas. La vía de nacimiento no fue un factor significativo ( $\times 2$ : 0.150 , valor $\mathrm{p}$ : 0.699), sin embargo la cesárea fue más frecuente (OR: 0.861). La valoración de Apgar al minuto y a los 5 minutos no fue una variable significativa. El uso de soluciones hiperosmolares no resultó ser significativa (x2: 0.228, valor p: 0.633, OR: 0.795$)$. El uso de ventilación mecánica no fue una variable significativa (x2: 1.662, valor p: 0.197, OR: 1.75). El uso de reanimación cardiopulmonar no fue una variable significativa (x2: 0.055 , valor $\mathrm{p}: 0.814, \mathrm{OR}: 0.895$ ). El uso de surfactante pulmonar no fue una variable significativa (x2: 0.892, valor $\mathrm{p}: 0.322$, OR: 1.643$)$.

Conclusión: Los factores asociados a hemorragia intraventricular son el peso al nacer menor o igual a $1500 \mathrm{~g}$, edad gestacional menor a 32 semanas y el sexo masculino, sin embargo la vía de nacimiento, la valoración de Apgar, el uso de soluciones hiperosmolares, el uso ventilación mecánica, el requerimiento de reanimación cardiopulmonar y uso de surfactante pulmonar no fueron factores de riesgo. El grado de Hemorragia intraventricular en neonatos prematuros fue el Grado I según la clasificación de Papile. (Horiz Med 2015; 15(2): 19-26)

Palabras clave: hemorragia intraventricular, neonatos prematuros. (Fuente: DeCS BIREME).

Factors associated with intraventricular hemorrhage in preterm infants at Hospital Regional Docente de Trujillo. December 2011 to December 2013.

\section{ABSTRACT}

Objective: To determine the factors associated with intraventricular hemorrhage in preterm newborns in "Hospital Regional Docente de Trujillo" in the period December 2011 to December 2013.

Material and Methods: Analytical, retrospective case-control study was conducted. The population consisted of 108 patients: 54 preterm infants with intraventricular hemorrhage and 54 preterm infants without intraventricular hemorrhage.

Results: Birth weight was found to be significant (x2: 6.438, p-value: 0.011$)$ reaching the rank of most frequent weight less or equal to 1500 . The sex of child proved to be a significant variable (x2: 6.366, p-value: 0.012$)$ being mostly males. Gestational age was found to be a significant variable $(\times 2: 10.394$, p-value: 0.001$)$ being the most common age less or equal to 32 Weeks. Birth type was not a significant factor (x2: 0.150, p-value: 0.699$)$, but caesarean section was more frequent (OR: 0.861$)$. The Apgar score at one minute and five minutes was not a significant variable. The use of hyperosmolar solutions was not found to be significant ( $\times 2$ : 0.228 , p-value: 0.633 , OR: 0.795$)$. The use of mechanical ventilation was not a significant variable $(\times 2$ : 1.662, $p$-value: 0.197 , OR: 1.75$)$. The use of cardiopulmonary resuscitation was not a significant variable $(\times 2: 0.055, p$-value: 0.814 , OR: 0.895$)$. The use of pulmonary surfactant was not a significant variable (x2: 0.892, p-value: 0.322, OR: 1.643). Conclusion: Factors associated with intraventricular hemorrhage are birth weight less than or equal to $1500 \mathrm{~g}$, gestational age less than or equal to 32 weeks and male gender of the newborn, but the type of birth, Apgar score, the use of hyperosmolar solutions, using mechanical ventilation, requirement of CPR and use of pulmonary surfactant was not a risk factor. The degree of intraventricular hemorrhage in preterm infants was the Grade I according to the classification of Papile. (Horiz Med 2015; 15(2): 19-26)

Key words: Intraventricular hemorrhage, preterm infants (Source: MeSH NLM).

1 Médico Cirujano de la Sanidad del Batallón de Selva N 85 Ampama, Amazonas. Perú

2 Médico Pediatra del Hospital Victor Lazarte Echegaray - Trujillo. Perú 


\section{INTRODUCCIÓN}

El principal tipo de hemorragia intracraneana observada en prematuros es la hemorragia intraventricular (HIV) ó hemorragia de la matriz germinal (HMG), cuya incidencia actual es el 15 - 20 $\%$ en los nacidos con edad gestacional menor de 32 semanas (1-3).

Unos doce mil neonatos prematuros desarrollan HIV cada año sólo en los Estados Unidos. La incidencia de HIV en recién nacidos de peso extremadamente bajo ( $<1000$ gramos) se ha reducido de $40-50 \%$ al $20 \%$ a finales de 1980; sin embargo, en las últimas dos décadas la ocurrencia se ha mantenido estacionaria $(4,5)$.

Se ha estimado que el $1.5 \%$ de todos los nacidos vivos en los Estados Unidos son prematuros, y que estos niños tienen un peso muy bajo al nacer (62.000 niños/año), el 36\% de los extremadamente prematuros (<25 semanas) desarrollan HIV grado III o IV, de los cuales, el $40 \%$ de estos pacientes requieren algún tratamiento para la hidrocefalia, se puede esperar aproximadamente 9,000 nuevos niños por año en los Estados Unidos con hidrocefalia consecuente a su prematuridad $(3,5,16)$.

Las manifestaciones clínicas de la hemorragia intraventricular en el prematuro varían desde cursar asintomáticamente, hasta un deterioro fulminante y la muerte; se describen tres tipos de evolución, una forma catastrófica, más frecuente en hemorragias graves y que progresa de minutos a horas, caracterizada por estupor o coma, apnea o hipoventilación, alteraciones en la homeostasis, hipotensión, baja de hematocrito, bradicardia, diabetes insípida o secreción inapropiada de hormona antidiurética.

Otra forma saltatoria, que progresa de horas a pocos días, mostrando deterioro de conciencia, reducción de la reactividad, hipotonía, movimientos oculares anormales, reducción del ángulo poplíteo y alteración del patrón respiratorio (apnea) ; una tercera forma evolutiva es clínicamente silente y puede caracterizar a $50 \%$ de los casos $(2,25,28)$.
En el prematuro el diagnóstico de la HMG/HIV se realiza mediante ultrasonografía transfontanelar (USG-TF) en tiempo real, se debe practicar a todos los recién nacidos con edad gestacional menor de 32 semanas, además se considerará realizar USGTF a neonatos mayores a 32 semanas con factores de riesgo para HIV como es asfixia perinatal o neumotórax, signos neurológicos anormales; existen dos clasificaciones para la HMG/HIV, la Clasificación de Papile describe 4 grados, siendo el Grado I: hemorragia aislada en la matriz germinal, sin presencia de hemorragia intraventricular, Grado II: hemorragia intraventricular sin dilatación ventricular, Grado III: HIV con dilatación ventricular, Grado IV: HIV con hemorragia parenquimatosa.

El engrosamiento del Epéndimo ha sido identificado por USG en los niños que tienen HIV relativamente pequeña sin lesión adyacente a la materia blanca hiperecóica, al contrario de ésta, el epéndimo es difícil identificar mediante USG en recién nacidos prematuros $(24,25)$.

El nacimiento prematuro es un factor de riesgo significativo para el desarrollo motor a largo plazo, la coordinación, desarrollo cognitivo y trastornos conductuales en los resultados de los sobrevivientes a HIV. El cerebelo ayuda a regular el tono muscular, la coordinación y la función cognitiva. Por lo tanto, los resultados adversos de la prematuridad pueden también resultar de disfunción del cerebelo.

El desarrollo del cerebelo se produce a partir de 4 semanas después de la concepción a 2 años después del nacimiento, lo que permite una gran ventana de alteraciones posibles (27).

Se ha demostrado que en recién nacidos con peso extremadamente bajo sobrevivientes a HIV grado IV tienen las mayores probabilidades de trastornos del neurodesarrollo en la edad de 18 a 24 meses hasta los 6 años de edad. Para cada grado de HIV, aumenta la probabilidad de deterioro neurológico de unilateral a bilateral, así como con el uso de esteroides y sepsis. Aunque la probabilidad de deterioro parece ser menor para el Grado II en comparación con Grado I no hay una diferencia significativa en las probabilidades de deterioro entre estos dos grados. Se asoció el sexo masculino 
en un $15.9 \%$, presentando HIV severa en un $38 \%$. Las mayores tasas de HIV severas fueron significativas en los grupos de pacientes con 1000 a 1499 grs.; se consideró como HIV Severa a los Grados III y IV de acuerdo a la Escala de Papile $(20,30)$.

Los glucocorticoides prenatales previenen la hemorragia de la masa germinal en los recién nacidos prematuros. Se ha observado en los materiales de autopsias humanas de los bebés prematuros, la cobertura de pericitos fue mayor en la matriz germinal de los bebés prematuros expuestos a glucocorticoides prenatales, en comparación con los niños no tratados. Se ha demostrado que el tratamiento con GC incrementa la cobertura de pericitos en la vasculatura de la masa germinal (18, 31).

El objetivo de este trabajo fue el analizar y determinar los factores asociados a hemorragia intraventricular en neonatos prematuros.

\section{MATERIAL Y MÉTODOS}

Estudio analítico de casos y controles, retrospectivo tomando como población a neonatos prematuros con diagnóstico de hemorragia intraventricular del Servicio de Neonatología del Hospital Regional Docente de Trujillo durante el periodo Diciembre 2011 a Diciembre 2013.

Se recopiló información de la base de datos del Servicio de Neonatología del HRDT para proceder con el estudio según tipo de muestreo aleatorio simple, tomando a 54 pacientes en el grupo de casos y 54 pacientes en el grupo de controles.

Criterios de inclusión: Neonatos prematuros con diagnóstico de hemorragia intraventricular confirmada por Ultrasonido transfontanelar del Servicio de Neonatología del HRDT durante los periodos Diciembre 2011 a Diciembre 2013.

Se excluyeron neonatos con muerte antes de las 24 horas de nacido, pacientes con otras malformaciones congénitas mayores del sistema nervioso central, displasias corticales, mielomeningocele, malformaciones arteriovenosas, otros tipos de sangrado del sistema nervioso central; que hayan sufrido trauma perinatal; neonatos con trastornos específicos de coagulación, tumores congénitos del SNC, retardo de crecimiento intrauterino (RCIU); que no cuentan con imagen de ecografía transfontanelar en su historia clínica; y prematuros cuya historia clínica estuviera incompleta.

Se revisó las historias clínicas de los pacientes seleccionados bajo los criterios de inclusión con previa autorización para su revisión y se localizaron los pacientes que tuvieron diagnóstico de Hemorragia Intraventricular. Se consultó en el documento si tienen los datos para las presentes variables: edad gestacional, peso al nacer, vía de nacimiento, maniobras de reanimación, Apgar al ler. y 5 ‥ Minuto de vida, días que requirieron ventilación mecánica, uso de soluciones hiperosmolares, grado de Hemorragia intracraneana de acuerdo a la Clasificación de Papile. Dichos datos se recolectaron en un formato, el cual nos sirvió para almacenarlos y estudiarlos para elaborar finalmente nuestros cuadros, gráficos y conclusiones.

Se utilizó para esto la prueba de chi cuadrado. Para el análisis respectivo se empleó el software SPSS v 18.0 para el manejo de la base de datos y procesamiento de la información. Se determinó el odds rattio para cada factor de riesgo para determinar si lo constituyen o no. Se considerará que hay significancia estadística si el valor de $p$ es $<0.05$.

\section{RESULTADOS}

El número de recién nacidos prematuros durante todo el periodo de investigación fue de 108 siendo de 54 los casos y 54 controles.

El peso al nacer resultó ser significativo (x2: 6.438; valor p: 0.011). Se estudiaron dos grupos: uno de prematuros con peso menor o igual a $1500 \mathrm{~g}$ los cuales fueron 29 (54\%) y de peso mayor a 1500 siendo 25 (46\%), obteniendo la mayor frecuencia en el primer grupo y un OR: 2.755. 
Tabla 1. Factores asociados a hemorragia intraventricular en neonatos prematuros.

\begin{tabular}{|c|c|c|c|c|c|}
\hline \multirow[t]{2}{*}{ Factores } & \multicolumn{2}{|c|}{ HIV } & \multirow[t]{2}{*}{ OR } & \multicolumn{2}{|c|}{ IC $95 \%$} \\
\hline & Si & No & & & \\
\hline \multicolumn{6}{|l|}{ Peso de recién nacido } \\
\hline$=1500 \mathrm{gr}$. & $29(54 \%)$ & $16(30 \%)$ & 2.755 & 1.248 & 6.083 \\
\hline $1500 \mathrm{gr}$. & $25(46 \%)$ & $38(70 \%)$ & & & \\
\hline \multicolumn{6}{|l|}{ Sexo } \\
\hline Masculino & $37(69 \%)$ & $24(44 \%)$ & 2.721 & 1.240 & 5.971 \\
\hline Femenino & $17(31 \%)$ & $30(56 \%)$ & & & \\
\hline Edad Gestacional & & & 3.909 & 1.670 & 9.149 \\
\hline-32 semanas & $43(80 \%)$ & $27(50 \%)$ & & & \\
\hline$>32$ semanas & $11(20 \%)$ & $27(50 \%)$ & & & \\
\hline \multicolumn{6}{|l|}{ Apgar al minuto } \\
\hline $3-5$ & $13(24 \%)$ & $11(20 \%)$ & 1.404 & 0.495 & 3.980 \\
\hline $6-7$ & $25(46 \%)$ & $24(44 \%)$ & 1.237 & 0.518 & 2.951 \\
\hline $8-9$ & $16(30 \%)$ & $19(35 \%)$ & & & \\
\hline \multicolumn{6}{|l|}{ Apgar a los 5 minutos } \\
\hline $3-5$ & $3(6 \%)$ & 0 & & & \\
\hline $6-7$ & $6(11 \%)$ & $11(20 \%)$ & 0.521 & 0.177 & 1.533 \\
\hline $8-9$ & $45(83 \%)$ & $43(80 \%)$ & & & \\
\hline \multicolumn{6}{|l|}{ Via de nacimiento } \\
\hline Cesárea & $29(54 \%)$ & $31(57 \%)$ & 0.861 & 0.403 & 1.840 \\
\hline Vaginal & $25(46 \%)$ & $23(43 \%)$ & & & \\
\hline Reanimación neonatal & & & 0.895 & 0.356 & 2.251 \\
\hline $\mathrm{Si}$ & $11(20 \%)$ & $12(22 \%)$ & & & \\
\hline No & $43(80 \%)$ & $42(78 \%)$ & & & \\
\hline Uso de Soluciones Hiperosmolares & & & 0.795 & 0.311 & 2.036 \\
\hline $\mathrm{Si}$ & $10(19 \%)$ & $12(22 \%)$ & & & \\
\hline No & $44(81 \%)$ & $42(78 \%)$ & & & \\
\hline Uso de ventilación mecánico & & & 1.75 & 0.744 & 4.117 \\
\hline $\mathrm{Si}$ & $18(33 \%)$ & $12(22 \%)$ & & & \\
\hline No & $36(67 \%)$ & $42(78 \%)$ & & & \\
\hline Uso de surfactante pulmonar & & & 1.643 & 0.612 & 4.411 \\
\hline $\mathrm{Si}$ & $12(22 \%)$ & $8(15 \%)$ & & & \\
\hline No & $42(78 \%)$ & $46(85 \%)$ & & & \\
\hline
\end{tabular}

El sexo del recién nacido resultó ser una variable significativa (x2: 6.366; valor $\mathrm{p}$ : 0.012 ) siendo en su mayoría el sexo masculino 37 casos $(69 \%)$ y con un OR: 2.721 frente al grupo de sexo femenino que resultó de 17 casos (31\%).

La edad gestacional resultó ser una variable significativa (x2: 10.394; valor $\mathrm{p}: 0.001)$ siendo más frecuente la edad menor o igual a 32 semanas 43 casos $(80 \%)$ con un OR: 3.909 , a diferencia de la edad mayor a 32 semanas las cuales fueron de 11 casos $(20 \%)$.

La vía de nacimiento no fue un factor significativo (x2: 0.150, valor $\mathrm{p}: 0.699$ ), sin embargo la cesárea fue más frecuente (OR: 0.861).
La valoración de Apgar al minuto y a los 5 minutos no fue una variable significativa.

El uso de soluciones hiperosmolares no resultó ser significativa (x2: 0.228 , valor $\mathrm{p}: 0.633$, OR: 0.795 ). El uso de ventilación mecánica no fue una variable significativa (x2: 1.662, valor p: 0.197, OR: 1.75).

El uso de reanimación cardiopulmonar no fue una variable significativa ( $\mathrm{x} 2: 0.055$, valor $\mathrm{p}: 0.814$, OR: 0.895). El uso de surfactante pulmonar no fue una variable significativa ( $\times 2$ : 0.892 , valor $\mathrm{p}$ : 0.322 , OR: 1.643). 


\section{DISCUSIÓN}

En este estudio se revisaron las historias clínicas de 54 recién nacidos prematuros que presentaron hemorragia intraventricular y sus respectivos controles, se observó una mayor proporción de HIV a menor peso al nacer, siendo que el peso menor de $1500 \mathrm{~g}$ presenta proporciones más altas de HIV (54\% versus $30 \%$ ) siendo dicho resultado estadísticamente significativo con un valor de P: 0.011y un OR: 2.755 , siendo éste junto al IC un valor mayor a 1 el cual nos refiere que se trata de un factor de riesgo. En relación a esto, los resultados del estudio de Cárdenas en el 2011 mostraron que a mayor peso existe un menor riesgo de desarrollar hemorragia intraventricular, hecho que coincide con lo reportado por Vergani y cols. en el 2000 y por Asha y cols. quienes presentaron una incidencia de hemorragia intraventricular asociada inversamente con el peso al nacer (39).

En cuanto al género, esta investigación determinó que el sexo masculino tiene mayor riesgo de presentar HIV (69\% versus 44\%) con relación de significancia estadística P: 0.012 y un valor de OR: 2.721 con un IC mayor a 1 , constituyendo un factor de riesgo. Mohamed $A$. y cols. demostraron dicha relación y concluye en que el nivel del estradiol y el sexo se asocian como un factor de protección en los recién nacidos del sexo femenino, por lo tanto hay una predominancia en la incidencia en varones (22). También en otro estudio realizado según Leyva en el 2008 se encuentra que hay predominancia en el sexo masculino obteniéndose $58 \%$ en relación a la frecuencia (35).

Se estudió también la edad gestacional, determinándose que a menor EG mayor riesgo de HIV. Se halló que los prematuros menores a 32 semanas presentan una mayor proporción de HIV $(80 \%$ versus $50 \%)$ con P: 0.001 , siendo estadísticamente significativo y un OR: 3.909 junto con el IC mayor a 1, interpretándose como un factor de riesgo. Este trabajo concuerda con el realizado por Vogtmann y cols. y Lee y cols. quienes encuentran mayor asociación en la aparición de hemorragia intraventricular para aquellos menores de 32 semanas (29). Según el estudio en el 2008 de Reyes, Leyva y Monzón concluyen que hay mayor asociación de aparición de hemorragia intraventricular para aquellos neonatos entre las 32 semanas de edad gestacional habiéndose hallado un $48 \%$ de un total de 263 neonatos (35).

Según la vía de nacimiento, no se observa que sea una variable con significancia estadística en el presente estudio y por lo tanto no es un factor asociado. Según nuestros hallazgos, la frecuencia de sujetos nacidos por parto vaginal con HIV (46\%) es similar a la presentada en aquellos carentes de HIV (43\%). Sin embargo este estudio se contrasta con lo hallado por Ayala en el 2007 en Colombia quien investigó de manera retrospectiva los factores de riesgo asociados a hemorragia intraventricular en prematuros menores de 32 semanas y por debajo de 1.500 gramos en una muestra de 330 historias clínicas; con aumento de la incidencia de hemorragia: parto vaginal $(p=0,004)$, atención del parto en centros de primer y segundo niveles $(p=$ $0,019)$ y la edad gestacional menor de 28 semanas $(p=0,032)(34)$.

No se demostró significancia estadísticamente significativa entre ninguna puntación del Score de Apgar, y la presencia de HIV, ni al minuto ni a los 5 minutos de vida respectivamente.

Esta relación concuerda con la mayoría de estudios internacionales. Según Roze y Jorien $M$, en su estudio determinan que tanto para la valoración de Apgar al minuto y a los 5 minutos no hay significancia estadística obteniendo además un OR: 0.79 e IC: $0.61-1.00$ (8).

Se realizó además el estudio de la frecuencia del grado de hemorragia intraventricular en nuestra muestra. Según la clasificación de Papile, se encontró 31 casos (57\%) de HIV Grado I, 18 casos (33\%) de HIV Grado II y 5 casos (9\%) de HIV Grado III. Ningún caso tuvo Grado IV. No toda la bibliografía internacional concuerda con los hallazgos en cuanto a la frecuencia del grado de HIV actualmente hallado, sin embargo Cervantes en el 2012 en México desarrollo una investigación con la finalidad de conocer los principales factores asociados a la hemorragia intraventricular en neonatos prematuros por medio de un estudio observacional retrospectivo en 1182 pacientes observando que la frecuencia de hemorragia fue de $6.1 \%$ y de acuerdo 
a la gravedad de la hemorragia intraventricular; el grado I ocurrió en el $62 \%$, grado II en $22 \%$, grado III en el $13 \%$ y en $3 \%$ grado IV (37).

Según el inicio de reanimación cardiopulmonar relacionado a hemorragia intraventricular no corresponde a un factor asociado. Se tuvo a solamente 11 pacientes $(20 \%)$ del grupo casos a quienes se les realizó RCP. De este pequeño grupo tanto a 5 pacientes se les realiza los pasos iniciales incluyendo aplicación de oxigeno libre, como a otros 5 pacientes que se les realiza ventilación de soporte refiriéndose a su mayoría en la aplicación de ventilación a presión positiva (VPP) con Ambu, y sólo 1 paciente recibió compresiones torácicas.

Sin embargo en el grupo control se encuentra 2 pacientes que requirieron de intubación orotraqueal, pero la causa se le atribuye a otro tipo de patologías. Este hallazgo concuerda con lo obtenido por Roze quien de 38 recién nacidos, 8 recibieron RCP $(p<0.005)$ y Vogtmann quien no encuentra significancia de dicha variable (8).

El uso de soluciones hiperosmolares en nuestro estudio no tuvo relación con la aparición de hemorragia intraventricular debido a que no hay significancia. Mercedes y cols en Panamá en el 2006 revisaron todos los expedientes de recién nacidos pretérminos menores de 37 semanas y con peso menor de 2500 gramos, con diagnóstico de Hemorragia Intraventricular; observando un mayor número de casos en los menores de 1500 gramos que usaron soluciones hiperosmolares, resultando chi cuadrado de 3.84 y OR mayor a 1 , siendo los intervalos de confianza bastante amplios $(p<0.05)$ (33).

El factor surfactante pulmonar fue requerido para mucho menos de la mitad de la población usando la dosis profiláctica siendo de 12 pacientes y resultando el $22 \%$. A ningún prematuro se le aplicó la dosis de rescate. Sin embargo el uso de surfactante pulmonar no corresponde a un factor de riesgo debido a que no se encuentra significancia estadística. Redondo en el 2006 en Argentina investigó los factores asociados a hemorragia intraventricular en 207 recién nacidos pretérmino nacidos consecutivamente; encontrando la administración de surfactante con un Odss ratio de $2.04(p<0.01)$.Se evidenció mayor incidencia y gravedad de las hemorragias a menor peso de nacimiento tomando como punto de corte un peso inferior a 1000 gramos se encontró con un Odss ratio de $2.57(\mathrm{p}<0.001)$ (32). Caro en el año 2010 en México publicó un estudio similar donde el uso surfactante pulmonar si fue un factor de riesgo para hemorragia intraventricular (OR: 2.32; IC 95\%: 1.05-5.14; $p=0.03)(36)$.

El uso de ventilación mecánica no corresponde a un factor asociado según el estudio, pero según su frecuencia se encontró que 18 pacientes fueron los que requirieron su soporte, siendo de 1 a 5 días de uso en el mayor de los casos, siendo 11 de los recién nacidos con HIV y representando el 61\%, disminuyendo la frecuencia conforme se prolonga el tiempo de días con apoyo ventilatorio. Según los hallazgos no hay asociación entre este factor con la aparición de HIV. Según Reyes, Leyva y Monzón se encontró un $67 \%$ de su población que emplea ventilación mecánica asistida, sin embargo p: $<0.005$ siendo no significativa (35).

Contrario a esto, Caro en el año 2010 en México encuentra que el uso de ventilación mecánica no fue un factor de riesgo para hemorragia intraventricular (OR: 3.60; IC 95\%: 1.46-8.91; $p=0.01$ ) (36).Lizama en el 2013 en Perú público un estudio con el objeto de determinar la incidencia y los factores de riesgo asociados a hemorragia intraventricular donde resultó significativo y por tanto un factor de riesgo el uso de ventilación mecánica (OR: 4.33, IC: 1.26-14.81, p:0.01) (38).

Finalmente concluimos que los factores asociados a hemorragia intraventricular son el peso al nacer menor o igual a $1500 \mathrm{~g}$, edad gestacional menor a 32 semanas y el sexo masculino, sin embargo la vía de nacimiento, la valoración de Apgar, el uso de soluciones hiperosmolares, el uso ventilación mecánica, el requerimiento de reanimación cardiopulmonar y uso de surfactante pulmonar no fueron factores de riesgo.

El grado de Hemorragia intraventricular en neonatos prematuros fue el Grado I según la clasificación de Papile. 


\section{REFERENCIAS BIBLIOGRÁFICAS}

1. John $\mathrm{P}$, et al. Hemorragia intracraneal y Leucomalacia Periventricular. Manual de Neonatología, Editorial Lippincott Williams \& Wilkins Año 2008;6ª . Edición.Cap.27 B, pág.493.

2. Gomella TL, et al. Hemorragia intracraneal. Neonatología. Editorial Panamericana. Año 2011; 6a. Edición. Cap. 96, pág. 479

3. Rennie J, et al. The baby who had an ultrasound as part of a preterm screening protocol. Neonatal Cerebral Investigation. Editorial Cambridge University Press. Año 2008; Lesson III, Chapter 8: Pág. 177.

4. Gmyrek D, et al. Risikoadjus- tierte Qualitätsbeurteilung am Beispiel der neonatalen Spätinfek- tion. Z Evid Fortbild Qual Gesundh Wesen 2011; 105: 124-32.

5. Andiman SE et al. The cerebral cortex overlying periventricular leukomalacia: Analysis of pyramidal neurons. Brain Pathol. 2010; 20:803-814.

6. Young Lee J, et al. Risk Factors for Periventricular-Intraventricular Hemorrhage in Premature Infants. J Korean Med. Sci.2010; 25:418-24.

7. Niwa T, et al. Punctate White matter lesions in infants: new insights using susceptibility -weighted imaging.Neuroradiology; 2011. 53: 669679.

8. Roze E, et al. Risk Factors for Adverse Outcome in Preterm Infants with Periventricular Hemorrhagic Infarction. Pediatrics. 2008; 122:e46.

9. Rees $\mathrm{S}$, et al. The biological basis of injury and neuroprotection in the fetal an neonatal brain. Int. J. Dev. Neurosci.2011; 29(6):551-63. 25.

10. O'Leary $\mathrm{H}$, et al. Elevated Cerebral Pressure Passivity is Associated with Prematurity-Related Intracranial Hemorrhage. Pediatrics.2009;124;302.

11. Merhar S, et al. Grade and Laterality of Intraventricular Hemorrhage to predict 18-22 Month Neurodevelopmental Outcomes in Extremely Low Birth Weight Infants.Acta Paediatr.2012;101(4):414-18.

12. Simard $M$, et al. Sulfonylurea receptor 1 in the germinal matrix of premature infants.Pediatr. Res.2008; 64(6):648-52.

13. Ballabh P, et al. Intraventricular Hemorrhage in Premature Infants: Mechanism of Disease. Pediatric Res.2010;67(1):1-8

14. Khan A, et al. Neonatal Incracraneal Ischemia and Hemorrhage:Role of Cranial Sonography and CT Scanning.J.Korean Neurosurg. 2010 Soc. 47:89-94.

15. Zubiaurre-Elorza L, et al. Cortical Thickness and Behavior Abnormalities in Children Born Preterm.2012;Vol.7:e42148.

16. Riva-Cambrin J, et al. Center Effect and Other factors influecing temporization and shunting of cerebrospinal fluid in preterm infants with intraventricular hemorrhage. J Neurosurg. Pediatr; 2012: 9 (5):473-481.

17. Gorm Greisen. Autoregulation of Cerebral Blood Flow. Neo Reviews.2007;1:22-30

18. Vinukonda G, et al. Effect of Prenatal Glucocorticoids on Cerebral Vasculature of the Developing Brain. Stroke; 2010, 41(8):1766-73
19. Schmidt B, et al. Effects of prophylactic indomethacin in Extremely low birth weight infants with and without adequate exposure to antenatal steroids. Arch.Pediatr. Adolesc. Med; 2011:165 (7)

20. Allen MC. Neurodevelopmental outcomes of preterm infants. Curr Opin Neurol. 2008; 21:123-128.

21. Roze E, et al. Functional outcome at school age of preterm infants with periventricular hemorrhagic infarction. Pediatrics 2009; 123:1493-1500.

22. Mohamed M, et al. Male Gender is Associated with Intraventricular Hemorrhage. Pediatrics; 2010:125:e333

23. Tuzcu V, et al. Altered Heart Rhythm Dynamics in Very Low Birth Weight Infants with Impending Intraventricular Hemorrhage. Pediatrics 2009; 123:810.

24. Adler I, et al. Mechanisms of Injury to White Matter Adjacent to a Large Intraventricular Hemorrhage in the Preterm Brain. J Clin Ultrasound; 2010:38(5) 254-258.

25. Hunt R, et al. Etamsilato para la Prevención de la Morbilidad y Mortalidad en Lactantes Prematuros ó con Muy Bajo Peso al Nacer. Cochrane Plus; 2011 Núm. 1 ISSN 1745-9990

26. Brouwer A et al. Neurodevelopmental outcome on preterm infants with severe intraventricular hemorrhage and therapy for posthemorrhagic ventricular dilatation. J Pediatr. 2008;152(5):648 654.

27. Tam E, et al. Differential Effects of Intraventricular Hemorrhage and White Matter Injury on Preterm Cerebellar Growth. J Pediatr; 2011:158 (3):366-371.

28. Garza S, et al. Hemorragia intracraneana. Neurología Neonatal; Programa de Actualización Continua en Neonatología, Año 2010 p.103-04

29. Vogtmann C, et al. Risk Adjusted Intraventricular Hemorrhage Rates in Very Premature Infants.Towards Quality Assurance Between Neonatal Units. Deutsches Ärzteblatt International / Dtsch Arztebl Int.2012; 109(31-32):527-33.

30. McCrea $\mathrm{H}$, et al. The Diagnosis, Mangement and Postnatal Prevention of Intraventricular Hemorrhage in the Preterm Neonate. Clin.Perinatol.2008;35 (4):777-VII.

31. Kim H, et al. Dexamethasone coordinately regulates angiopoietin-1 and vegf: A mechanism of glucocorticoid-induced stabilization of blood-brain barrier. Biochem Biophys Res Commun 2008; 372:243-248.

32. Redondo O, Rodríguez A. Hemorragia intracraneana del prematuro. Frecuencia de presentación y factores de riesgo. Arch Argent Pediatr 2006; 101(4): 56 - 59

33. Mercedes $\mathbf{M}$, Moreno $\mathrm{M}$. Factores de Riesgo asociados a la Hemorragia Peri/Intraventricular en pacientes prematuros menores de 2500 gramos. Revista Pediátrica de Panama.2006, 35:22-30.

34. Ayala A, Carvajal L. Evaluación de la incidencia y los factores de riesgo para hemorragia intraventricular (HIV) en la cohorte de recién nacidos prematuros atendidos en la unidad neonatal del Hospital Universitario San Vicente de Paúl, de Medellín. En el periódo comprendido entre Enero 1999 y Dic 2004 latreia: 2007; 20 (4): 341-353. 
35. Reyes - Gutiérrez E, Dautt-Leyva J, Monzón-Ruelas A. Hemorragia Intracraneal en Recién Nacidos Pretérmino Menores de 2,000 g: A S S in 2008; 2 (3):92-97.

36. Caro A, Barrera J. Evaluacion de factores prenatales, perinatales y posnatales para hemorragia intraventricular en prematuros menores de 33 semanas de edad gestacional. Gaceta Médica de México. 2010; (2):146-151.

37. Cervantes M, Rivera M, Villegas R. Hemorragia intraventricular en recién nacidos pretérmino en una Unidad de Tercer Nivel en la Ciudad de México. Perinatol Reprod Hum 2012; 26 (1): 17-24.

38. Lizama 0 . Incidencia y factores asociados a la hemorragia intraventricular en prematuros de muy bajo peso en el Hospital Nacional Guillermo Almenara Irigoyen. Tesis 2013. Universidad Cayetano Heredia

39. Cardenas J, Florez B. Factores asociados al desarrollo de HIV en neonatos pretermino en el Hopital Occidente de Kennedy. Tesis 2011. Universidad Militar de Nueva Granada.

\section{Fuentes de financiamiento}

El estudio ha sido financiado por el autor.

\section{Conflicto de interés}

El autor declara no tener ningún conflicto de interés.

\section{Correspondencia:}

Glauco Valdivieso Jiménez

Dirección: Mz. B Lote 4 Urb. UPAO II - Trujillo

Teléfono: 969753278

Correo: glaucovald@gmail.com

\title{
HORIZONTE MÉDICO
}

Indizada a: Scielo, Latindex, DOAJ , entre otras

\section{Difundiendo la investigación, más allá; de nuestras fronteras}

\author{
Se distribuye gratuitamente y por canje. \\ Está disponible a texto íntegro en el siguiente \\ e-mail: horizonte_medico@usmp.pe y \\ en la web: \\ www. medicina. usmp. edu. pe/ horizonte/ principal. php
}

Síguenos en:

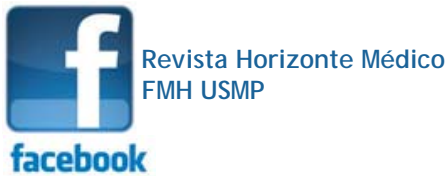

@HorizMed

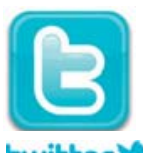

twittery 